\title{
Poly-L-Lactic Acid Increases Collagen Gene Expression and Synthesis in Cultured Dermal Fibroblast (Hs68) Through the p38 MAPK Pathway
}

\author{
Sung-Ae Kim, Hyo-Seon Kim, Jin-Woong Jung, Sung-Il Suh ${ }^{1}$, Young-Wook Ryoo \\ Departments of Dermatology and ${ }^{1}$ Microbiology, School of Medicine \& Institute for Medical Science, Keimyung University, Daegu, Korea
}

\begin{abstract}
Dear Editor:
Demand for new biomaterials that produce good results in clinical fields requiring tissue restoration and replacement is expected to continue to increase ${ }^{1}$. Poly-L-lactic acid (PLLA) has been widely used since it was approved by the Food and Drug Administration (U.S. FDA) as an injectable facial volumizer in the treatment of lipoatrophy due to its significant therapeutic effectiveness ${ }^{2}$. However, except for those clinical and histological reports, few data on the molecular biologic effects of PLLA have been reported ${ }^{3}$. In each of these studies, PLLA-induced mild inflammation and the paracrine effects of foreign body giant cells are presumed to be the central mechanistic feature, resulting in increases in the production of new collagen over the months. However, the exact mechanisms of how PLLA can increase collagen production are still unknown. Based on our clinical experiences, the facial volume-augmenting effect of PLLA was noticeable even at one month after injection, which is much faster than the rate reported in papers; thus, we hypothesized that PLLA could directly affect dermal fibroblasts ${ }^{3,4}$. The goal of this paper was to assess the effect of PLLA on collagen synthesis and related signal pathways in cultured dermal fibroblast.

Hs68 (human dermal fibroblast) and cell line were cultured and stimulated with PLLA. Vials of dry powder of PLLA (Sculptra ${ }^{\circledR}$; Sanofi Aventis, Paris, France) were used in the present study. Five milliliters of sterile water was added to the dry powder of Sculptra ${ }^{\circledR}$ and finally adjusted
\end{abstract}

to $0.1 \%$ concentration in the culture medium ${ }^{5}$. Type I collagen gene expression induced by PLLA was evaluated using real time RT-PCR, and we performed ELISA and Western-blot analyses to confirm the effect of PLLA on type I collagen synthesis.

The dramatically increasing effects of PLLA on type I collagen gene expression were detected using real time RT-PCR at 48-hours incubation, suggesting that PLLA increased transcription of type I collagen gene within a relatively shorter period of time than expected (Fig. 1A). To examine whether the increase in collagen mRNA was followed by an increase in collagen protein synthesis, we measured the procollagen concentration in the medium according to the manufacturer's protocol (Takara Bio, Otsu, Japan). Consistent with the data on collagen gene expression, the amounts of procollagen were significantly increased by PLLA (Fig. 1B). In the Western blot analysis, treatment of $\mathrm{Hs} 68$ with $0.1 \%$ PLLA resulted in a significant increase in type I collagen in 48 hours (Fig. 1C, D). To dissect the signal transduction pathways involved in the up-regulation of type I collagen production, various signal molecules were tested (Supplementary Fig. 1). Only the anti-phospho p38 mitogen-activated protein kinase (p38 MAPK), JNK, and Akt antibodies were detected specifically as the phosphorylated form of p38, Akt, and JNK, respectively, after the PLLA treatment (Fig. 2A, B). Thus, we focused on the functional role of those molecules in the up-regulatory effect of PLLA. Pre-incubation of Hs68 cell

\footnotetext{
Received January 29, 2018, Revised February 19, 2018, Accepted for publication March 27, 2018
}

Corresponding author: Young-Wook Ryoo, Department of Dermatology, School of Medicine \& Institute for Medical Science, Keimyung University, 56 Dalseong-ro, Jung-gu, Daegu 41931, Korea. Tel: 82-53-250-7624, Fax: 82-53-250-7626, E-mail: ryoo111@dsmc.or.kr ORCID: https://orcid.org/0000-0002-2477-6263

This is an Open Access article distributed under the terms of the Creative Commons Attribution Non-Commercial License (http://creativecommons.org/ licenses/by-nc/4.0) which permits unrestricted non-commercial use, distribution, and reproduction in any medium, provided the original work is properly cited.

Copyright (c) The Korean Dermatological Association and The Korean Society for Investigative Dermatology 

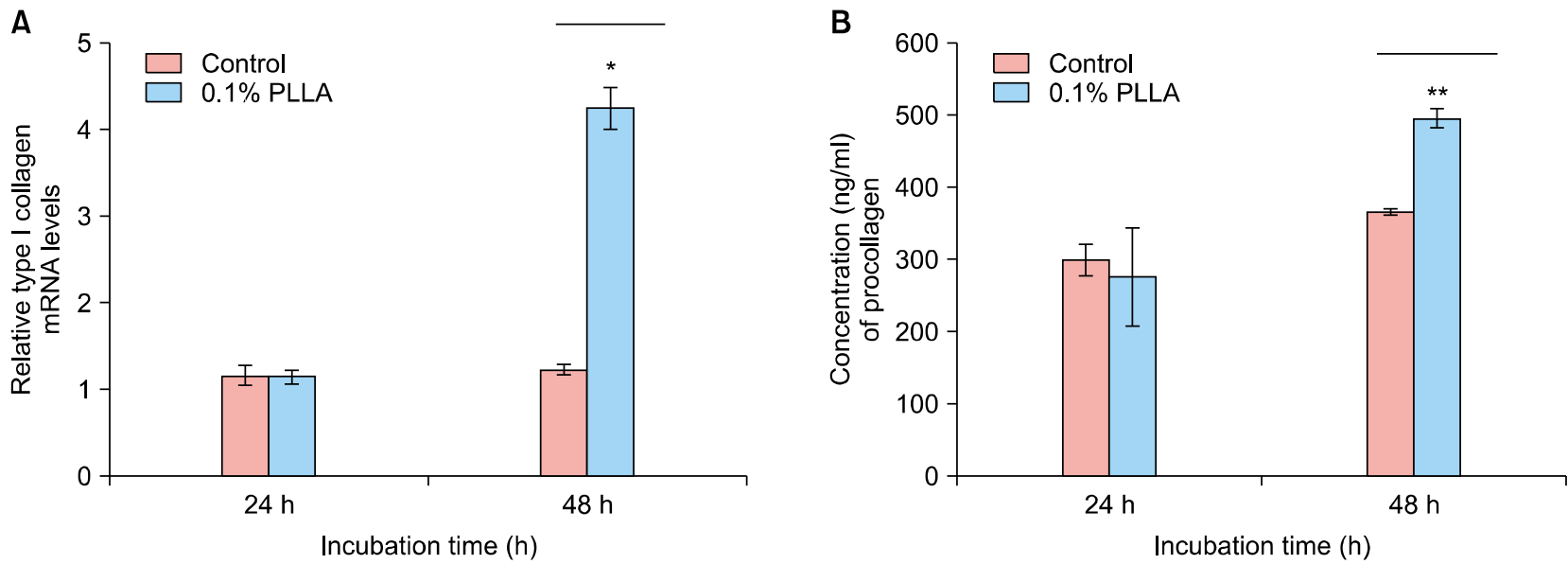

C

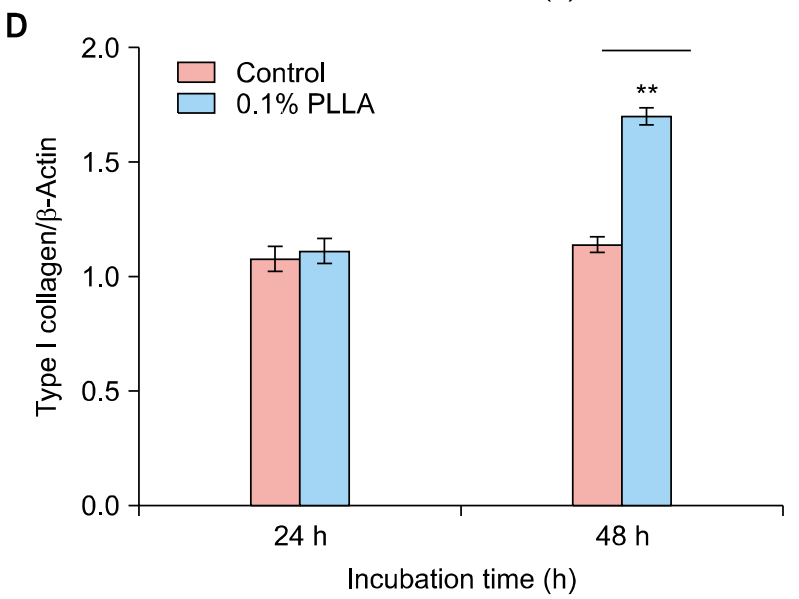

Fig. 1. Effect of PLLA on collagen synthesis. (A) Human fibroblast cell line (Hs68) was treated with PLLA at $0.1 \%$ concentration for 24 and 48 hours. Type I collagen expression was determined using real time RT-PCR. The relative levels of mRNA were normalized against GAPDH levels from the same cDNA preparation. Data represent the mean \pm SD of the results in three independent experiments. ${ }^{*} p<0.05$. (B) The levels of type I procollagen protein were determined using ELISA for 24 and 48 hours. Data represent the mean \pm SD of the results in three independent experiments. ${ }^{* *} p<0.05$. (C) The levels of type I collagen protein were determined using the Western blot analysis for 24 and 48 hours. (D) The relative levels of type I collagen protein were normalized against beta-actin levels from the same protein preparation. Data represent the mean \pm SD of the results in three independent experiments. ${ }^{* *} p<0.01$, PLLA: poly-L-lactic acid, RT-PCR: reverse transcription-polymerase chain reaction, GAPDH: glyceraldehyde 3-phosphate dehydrogenase, SD: standard deviation, ELISA: enzyme-linked immunosorbent assay.

with SB203580 (p38/Akt inhibitor) and SP600125 (JNK inhibitor) resulted in significant inhibition of PLLA-induced collagen synthesis (52\% and $42 \%$, respectively) (Fig. 2C, D). Together, these data demonstrated that PLLA has a direct stimulatory effect on collagen production, and these results were mediated through the activation of the signaling proteins of $\mathrm{p} 38, \mathrm{Akt}$, and JNK.

It has been reported that one of the most important characteristics of biomaterials is their ability to trigger adequate levels of host inflammatory responses. Therefore, designing biomaterial-based therapies must be accompanied by biomaterial-immune system interactions ${ }^{6}$. PLLA has become one of the most promising biomaterials because of its advantages, such as biocompatibility, bio- degradability, and minimal inflammatory inductive property $^{4}$. PLLA is superior to conventional classic fillers in terms of stability and effect persistence; thus, it is thought that not only the face but also any part of the body will be used more as a supplement to fill tissue defects ${ }^{3}$. At the sites where the PLLA was administered, a weak inflammatory reaction, infiltration of foreign body giant cells, and elongated fibroblasts were observed, which are presumed to be the central mechanistic features ${ }^{4}$. Type I collagen statistically and significantly increased from baseline to 6 months after PLLA injection in human $\mathrm{skin}^{7,8}$. Moreover, the clinically volume-augmenting effect of PLLA could be confirmed even within one month after injection, which is much faster than the rate reported in an- 
A

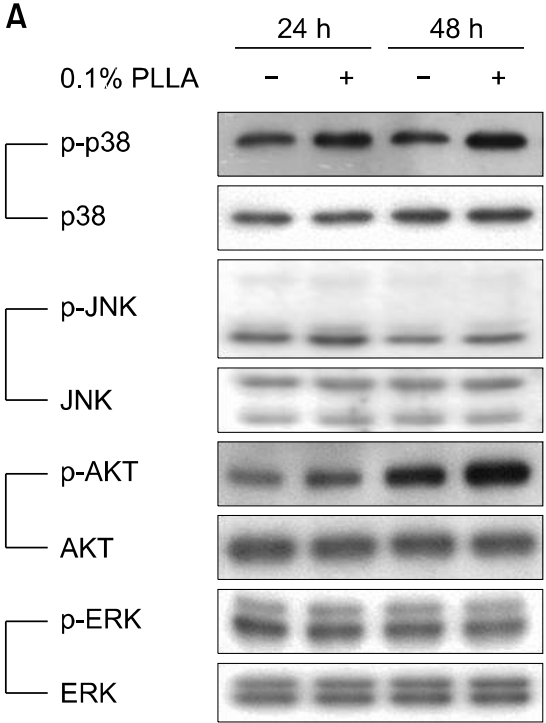

C

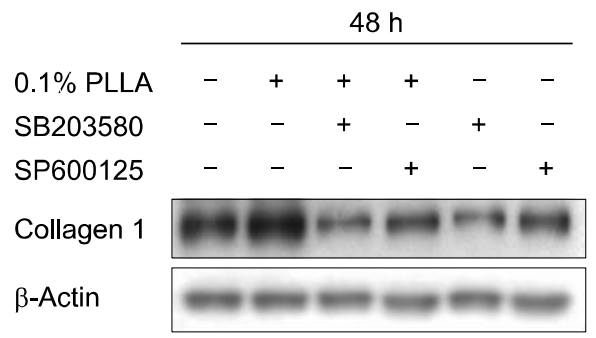

B
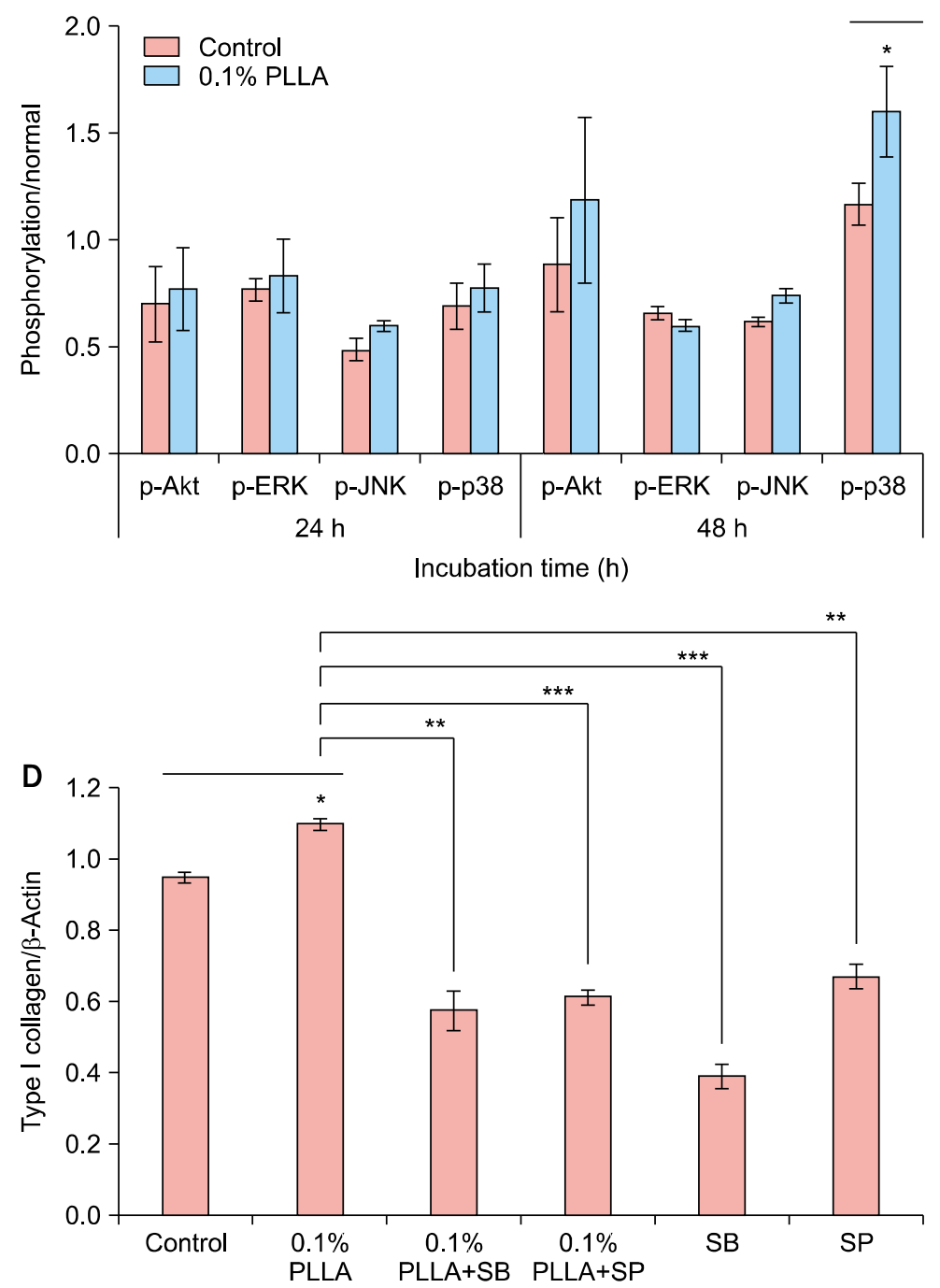

Fig. 2. (A) Activation of p38, JNK, and Akt is critical for PLLA-induced collagen I gene expression. Human dermal fibroblasts (HS68) were treated with PLLA $(0.1 \%)$ for 24 and 48 hours. At each time, cell lysates were prepared and used for type I collagen, p-p38 MAPK, p38 MAPK, p-JNK, JNK, p-Akt, Akt, p-ERK, and ERK Western blot analyses with respective antibodies. (B) Data represent the mean \pm SD of the results in three independent experiments. ${ }^{*} p<0.05$. (C) Effects of MAPK inhibitors on PLLA-induced type I collagen production. Hs68 cells were pretreated with the indicated concentration of SB203580 (p38/Akt inhibitor) and SP600125 (JNK inhibitor) for 30 minutes and treated with $0.1 \%$ PLLA for an additional 48 hours. Cell lysates were prepared and used for p-p38 MAPK, p-JNK, p-Akt, p-ERK, and Western blot analyses with respective antibodies. (D) Data represent the mean \pm SD of the results in three independent experiments. ${ }^{*} p<0.05,{ }^{* *} p<0.01,{ }^{* * *} p<0.001$. PLLA: poly-L-lactic acid, p-: phosphorylated, SD: standard deviation, MAPK: mitogen-activated protein kinase, SB: SB203580 (p38/Akt inhibitor), SP: SP6001.

other study ${ }^{8}$. Therefore, it is assumed that there is another mechanism of collagen synthesis that enables early volumizing results. In this study, we have found that PLLA directly acts on dermal fibroblasts leading to a significant increase in type I collagen gene expression and protein synthesis. In addition, those reactions already began at 48-hours incubation and signal pathways through p38, Akt, and JNK were involved. These results supported by previously published reports suggest that MAPK and Akt pathways are involved in polymer-induced collagen synthesis. Ren et al. ${ }^{9}$ reported that both MAPK and Akt signaling pathways were activated by hydroxyapatite and PLLA, but its precise mechanisms of action remain to be elucidated. The Akt signaling pathway is involved in fibroblast migration and promotes collagen synthesis, resulting in cutaneous wound contraction ${ }^{10}$.

Our results provide the first evidence that PLLA directly stimulates dermal fibroblasts to increase collagen synthesis 
through activation of the p38, Akt, and JNK signaling pathways might be influenced by either an adjacent macrophage, which may serve as a late trigger of collagen synthesis or a limitation of the in vitro condition. However, it must be noted that the present study is limited in providing insights into the collagen synthesis effects of PLLA in an artificial in vitro system. As collagen synthesis is a complex in vivo event, many questions remain to be addressed. These in vitro data nevertheless provide a concept that may allow insights into the biologic effect of PLLA on fibroblast. Furthermore, P38, Akt, and JNK are signal transduction molecules that regulate cell growth, differentiation, and apoptosis. Hence, further research about the effects of PLLA on other extracellular matrix components is needed in the future.

\section{ACKNOWLEDGMENT}

This work was supported by the research promoting grant from Keimyung University Dongsan Medical Center 2016.

\section{SUPPLEMENTARY MATERIALS}

Supplementary data can be found via http://anndermatol. org/src/sm/ad-31-97-s001.pdf.

\section{CONFLICT OF INTEREST}

The authors have nothing to disclose.

\section{ORCID}

Sung-Ae Kim, https://orcid.org/0000-0002-6040-6630 Hyo-Seon Kim, https://orcid.org/0000-0002-1274-1055 Jin-Woong Jung, https://orcid.org/0000-0001-7592-3035 Sung-II Suh, https://orcid.org/0000-0003-2078-292X
Young-Wook Ryoo, https://orcid.org/0000-0002-2477-6263

\section{REFERENCES}

1. Sridharan R, Cameron AR, Kelly DJ, Kearney CJ, O'Brien FJ. Biomaterial based modulation of macrophage polarization: a review and suggested design principles. Mater Today 2015; 18:313-325.

2. Vleggaar D, Bauer U. Facial enhancement and the European experience with Sculptra (poly-l-lactic acid). J Drugs Dermatol 2004;3:542-547.

3. Stein P, Vitavska O, Kind P, Hoppe W, Wieczorek H, Schürer NY. The biological basis for poly-L-lactic acid-induced augmentation. J Dermatol Sci 2015;78:26-33.

4. Vleggaar D, Fitzgerald R, Lorenc ZP. Composition and mechanism of action of poly-L-lactic acid in soft tissue augmentation. J Drugs Dermatol 2014;13(4 Suppl):s29-31.

5. Courderot-Masuyer C, Robin S, Tauzin $H$, Humbert $P$. Evaluation of the behaviour of wrinkles fibroblasts and normal aged fibroblasts in the presence of poly-L-lactic acid. J Cosmet Dermatol Sci Appl 2012;2:20-27.

6. Correia CR, Gaifem J, Oliveira MB, Silvestre R, Mano JF. The influence of surface modified poly(l-lactic acid) films on the differentiation of human monocytes into macrophages. Biomater Sci 2017;5:551-560.

7. Lemperle G, Morhenn V, Charrier U. Human histology and persistence of various injectable filler substances for soft tissue augmentation. Aesthetic Plast Surg 2003;27:354-366.

8. Goldberg D, Guana A, Volk A, Daro-Kaftan E. Single-arm study for the characterization of human tissue response to injectable poly-L-lactic acid. Dermatol Surg 2013;39:915-922.

9. Ren Y, Ni Z, Wang Y, Zhang L, Liu G, Liu X. 10\% HA/ SPEEK/PEEK composite promotes proliferation and differentiation of osteoblast cells through the MAPK and PI3K/AKT signaling pathways. Int J Clin Exp Med 2017;10:2127-2136.

10. Li G, Li YY, Sun JE, Lin WH, Zhou RX. ILK-PI3K/AKT pathway participates in cutaneous wound contraction by regulating fibroblast migration and differentiation to myofibroblast. Lab Invest 2016;96:741-751. 


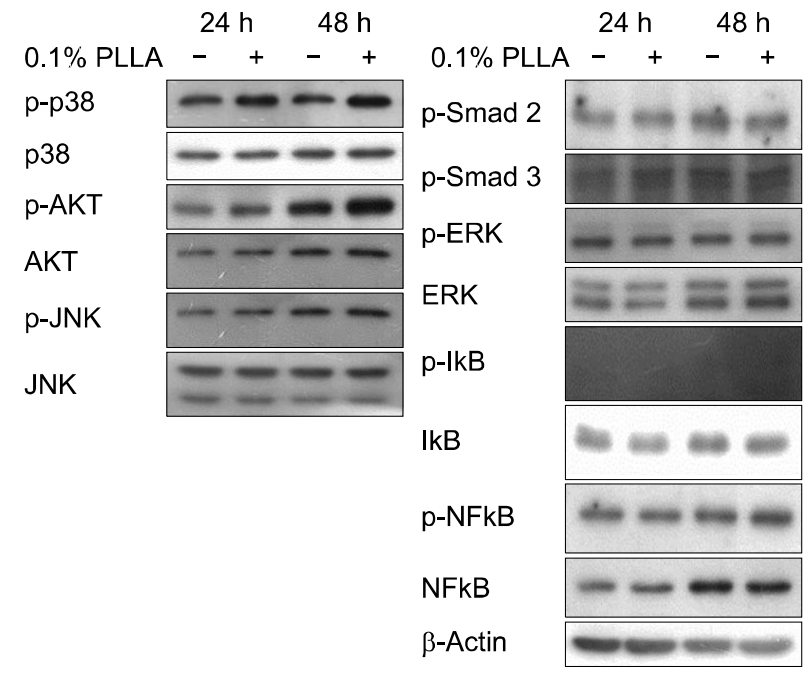

Supplementary Fig. 1. Effect of PLLA on collagen synthesis in collagen synthesis in human dermal fibroblast (HS68). Cells were treated with $01 \%$ PLLA for 24 and 48 hours. The various signal molecules were determined using Western blotting. Activation of p38, JNK, and Akt is critical for PLLA-induced collagen I gene expression. At each time, cell lysates were prepared and used for p-p38 MAPK, p38 MAPK, p-JNK, JNK, p-Akt, Akt, p-ERK, and ERK Western blot analyses with respective antibodies. PLLA: poly-L-lactic acid, p-: phosphorylated. 